\title{
The Role of Neurotrophic Factors Conjugated to Iron Oxide Nanoparticles in Peripheral Nerve Regeneration: In Vitro Studies
}

\author{
Ofra Ziv-Polat, ${ }^{1}$ Abraham Shahar, ${ }^{1}$ Itay Levy, ${ }^{2}$ Hadas Skaat, ${ }^{2}$ Sara Neuman, ${ }^{1}$ \\ Federica Fregnan, ${ }^{3}$ Stefano Geuna, ${ }^{3}$ Claudia Grothe, ${ }^{4,5}$ Kirsten Haastert-Talini, ${ }^{4,5}$ \\ and Shlomo Margel ${ }^{2}$ \\ ${ }^{1}$ NVR Research Ltd., Heharash Street 11, 74031 Ness-Ziona, Israel \\ ${ }^{2}$ Institute of Nanotechnology and Advanced Materials, Department of Chemistry, Bar-Ilan University, 52900 Ramat-Gan, Israel \\ ${ }^{3}$ Department of Clinical and Biological Sciences, Università degli studi di Torino, Regione Gonzole 10, 10043 Orbassano, Italy \\ ${ }^{4}$ Institute of Neuroanatomy, Hannover Medical School, Carl-Neuberg-Street 1, 30623 Hannover, Germany \\ ${ }^{5}$ Center for Systems Neuroscience (ZSN) Hannover, 30559 Hannover, Germany
}

Correspondence should be addressed to Ofra Ziv-Polat; ofraziv1@yahoo.com

Received 9 February 2014; Accepted 17 June 2014; Published 16 July 2014

Academic Editor: Fausto Viterbo

Copyright (C) 2014 Ofra Ziv-Polat et al. This is an open access article distributed under the Creative Commons Attribution License, which permits unrestricted use, distribution, and reproduction in any medium, provided the original work is properly cited.

Local delivery of neurotrophic factors is a pillar of neural repair strategies in the peripheral nervous system. The main disadvantage of the free growth factors is their short half-life of few minutes. In order to prolong their activity, we have conjugated to iron oxide nanoparticles three neurotrophic factors: nerve growth factor $(\beta N G F)$, glial cell-derived neurotrophic factor (GDNF), and basic fibroblast growth factor (FGF-2). Comparative stability studies of free versus conjugated factors revealed that the conjugated neurotrophic factors were significantly more stable in tissue cultures and in medium at $37^{\circ} \mathrm{C}$. The biological effects of free versus conjugated neurotrophic factors were examined on organotypic dorsal root ganglion (DRG) cultures performed in NVR-Gel, composed mainly of hyaluronic acid and laminin. Results revealed that the conjugated neurotrophic factors enhanced early nerve fiber sprouting compared to the corresponding free factors. The most meaningful result was that conjugated-GDNF, accelerated the onset and progression of myelin significantly earlier than the free GDNF and the other free and conjugated factors. This is probably due to the beneficial and long-acting effect that the stabilized conjugated-GDNF had on neurons and Schwann cells. These conclusive results make NVR-Gel enriched with conjugated-GDNF, a desirable scaffold for the reconstruction of severed peripheral nerve.

\section{Introduction}

In case of peripheral nerve injuries with long distance defects (gaps), autologous nerve grafting is the treatment of choice. However, the availability of autologous nerve grafts is limited especially when a large amount of grafting material is needed, because it requires sacrifices of healthy nerves [1]. Therefore, many efforts are being made to develop artificial nerve grafts. The artificial nerve grafts are usually composed of conduits, which guide the regenerating axons to the distal nerve stump, filled with biomaterials decorated with neurotrophic factors (neuronal growth factors) [2].

Neurotrophic factors are signaling proteins which support neural survival and axonal growth. In addition, neurotrophic factors play a significant role in the maintenance of neuronal function throughout an entire lifetime. There are a variety of neurotrophic factors that have been identified and studied at various levels from molecular interactions to macroscopic tissue responses (reviewed in [3-6]). In the 
present study we focused on three neurotrophic factors: nerve growth factor $(\beta \mathrm{NGF})$, glial cell-derived neurotrophic factor (GDNF), and basic fibroblast growth factor (FGF-2).

NGF (discovered in the early 1950s by Rita LeviMontalcini who won the 1986 Nobel Prize in Medicine for this discovery [7]) is a polypeptide composed of three subunits alpha, beta, and gamma. Only the beta subunit $(\beta N G F)$ is responsible for the physiological activity ascribed to NGF, and it is active in the absence of the other two subunits [8]. $\beta$ NGF is vital for the development and maintenance of both central and peripheral nervous system neurons. It is known to regulate the growth and differentiation of sympathetic and sensory neurons. In addition, $\beta$ NGF plays a key role in nerve injury repair and in preventing neuronal degeneration (reviewed in $[3,9]$ ).

GDNF has been demonstrated to enhance the survival and outgrowth of motor neurons as well as sensory neurons [3]. It also promotes in vivo myelination of unmyelinated nerve fibers $[10,11]$. This factor was also found to act on dopaminergic neurons in the substantia nigra, whose death is responsible for Parkinson's disease [12, 13].

FGF-2 is a potent mitogenic polypeptide which induces cell divisions in a variety of mesodermal cell types and cells of neuroectodermal origin (reviewed in [14, 15]). FGF2 has been shown to promote neuron survival, spinal cord regeneration, peripheral nerve regeneration, and neuritogenesis of motor neurons [16-19]. FGF-2 also stimulates the proliferation and differentiation of neuronal precursor cells from mouse and human olfactory epithelium [3, 14, 20-22] as well as of Schwann cells [23].

Because of their positive effect on neurons, neurotrophic factors are attractive candidates for therapy of acute injuries to the nervous system (such as reconstruction of peripheral nerve after a severe segment loss, especially in cases of a long distance nerve defect) as well as chronic neurodegenerative diseases (such as Alzheimer's, Huntington's, and Parkinson's disease) (reviewed in $[3,4]$ ). Also, in laboratory research, it is common to add neurotrophic factors to cell cultures to enhance neuronal cell regeneration. However, the main disadvantage of free growth factors is their short in vivo halflife time due to rapid enzymatic degradation, which leads to the loss of their biological activity after a short period of time [24]. For example, the half-life time of FGF-2, brain derived neurotrophic factor (BDNF), and $\beta$ NGF in blood is 1.5-3, 10 , and $30 \mathrm{~min}$, respectively [25-29]. In order to prolong their activity and bioavailability, it has been suggested to conjugate the growth factors onto or encapsulated within nanostructures of diverse types of synthetic and natural materials $[24,30]$.

In the present study, iron oxide nanoparticles were used for neurotrophic factor conjugation. These nanoparticles provide magnetic properties, high surface-area-to-volume ratio, biocompatibility, and biodegradability. Iron oxide magnetic nanoparticles are considered to be nontoxic and are already being used for various biomedical applications, such as diagnostics, cell labeling and sorting, DNA separation, MRI and X-ray contrast agents, gene and drug delivery, and hyperthermia (reviewed in $[21,22]$ ). The magnetic properties of these nanoparticles may also be used for targeting of a drug immobilized on magnetic materials under the action of an external magnetic field [31]. In addition, the magnetic properties of the iron oxide nanoparticles enable their purification from excess reagents, during the synthesis process, easily by using magnetic columns.

The aim of the present study was to examine in vitro the stability and the biological effects of $\beta$ NGF, GDNF, and FGF-2 conjugated covalently to iron oxide nanoparticles, with the prospective to use them in artificial conduits for peripheral nerve reconstruction. Our studies were conducted on cultures of organotypic dorsal root ganglion (DRG) seeded in NVR-Gel [32] as a possible hydrogel scaffold for filling artificial conduit [2].

\section{Materials and Methods}

2.1. Synthesis of Iron Oxide Nanoparticles. Magnetic iron oxide nanoparticles were prepared in two main ways as follows.

(a) Gelatin coated iron oxide nanoparticles containing the fluorescent probe rhodamine of $15.0 \pm$ $1.4 \mathrm{~nm}$ dry diameter were prepared by nucleation and then controlled the growth of iron oxide layers onto rhodamine isothiocyanate (RITC) conjugated gelatin/iron oxide nuclei, according to our previous publications [21, 33].

(b) Dextran coated iron oxide magnetic nanoparticles of $9.5 \pm 0.9 \mathrm{~nm}$ dry diameter were prepared as described previously by Molday and Mackenzie [34].

2.2. Conjugation of Neural Growth Factors to the Iron Oxide Nanoparticles. Covalent conjugation of $\beta \mathrm{NGF}$, GDNF, or FGF-2 (PeproTechAsia, Israel) to the surface of the iron oxide nanoparticles (each type individually) was performed as described previously [21, 22, 33]. Briefly, the iron oxide nanoparticles coated either with gelatin (which provides hydroxyl and primary amine functional groups) or with dextran (which provides hydroxyl groups) were functionalized with activated double bonds by interacting the functional groups of the gelatin or dextran with excess divinyl sulfone (DVS) via the Michael addition reaction. The residual activated double bonds were then used for covalent binding of the different neurotrophic factors, again via the Michael addition reaction. The concentration of each neurotrophic factor conjugated to the nanoparticles was determined by using the appropriate ELISA Kit (PeproTech Asia, Israel, and Boster Immunoleader, USA).

\subsection{Comparative Stability Studies of Free versus Conjugated Neurotrophic Factors}

(a) Stability in Cell Cultures. Free or conjugated neurotrophic factors were added once to the medium of dissociated DRG cultures (final concentration $10 \mathrm{ng} / \mathrm{mL}$, each factor). The cultures were placed in a $5 \% \mathrm{CO}_{2}$ incubator at $37^{\circ} \mathrm{C}$ for 9 days. The culture medium was not changed during the entire period of 
the experiment, and aliquots from it were collected at different points in time after cultivation. The concentrations of the residual factors in the aliquots were measured using the appropriate ELISA Kit.

(b) Stability at $37^{\circ} \mathrm{C}$ in Medium. Samples of culture media containing free or conjugated neurotrophic factors (final concentration $10 \mathrm{ng} / \mathrm{mL}$, each factor) were kept at $37^{\circ} \mathrm{C}$. Aliquots were collected at different time points, and the concentration of the residual free and conjugated neurotrophic factors in the samples was measured as described above.

2.4. Culture Medium. The culture medium was prepared from $90 \%$ Dulbecco's modified eagle medium-nutrient mixture F-12 (DMEM-F12), 10\% heat-denatured fetal calf serum (FCS), $6 \mathrm{~g} / \mathrm{L}$ D-glucose, $2 \mathrm{nM}$ glutamine, $25 \mu \mathrm{g} / \mathrm{mL}$ gentamycin, and $50 \mathrm{ng} / \mathrm{mL}$ IGF-I (all purchased from Biological Industries, Israel).

2.5. NVR-Gel for Neuronal Cultivation. NVR-Gel (NVR Labs proprietary [32]) is composed of two main components: high molecular hyaluronic acid $\left(\mathrm{HA}, 3 \times 10^{6} \mathrm{Da}\right)$ and laminin, both known to be inherent elements of the extracellular matrix. For cell cultivation, HA of 1\% (BTG Polymers, Israel) was diluted with culture medium to the concentrations of $0.3-$ $0.5 \%$. Laminin (Sigma) was then mixed with the diluted $\mathrm{HA}$ to a final concentration of $10 \mathrm{ng} / \mathrm{mL}$. Free or conjugated neurotrophic factors ( $\beta$ NGF, GDNF, and FGF-2) were finally added, separately or together (10 $\mathrm{ng} / \mathrm{mL}$ of each factor), to complete the NVR-Gel composition.

2.6. Preparation of Organotypic DRG Cultures. All the experiments were carried out and authorized by the local ethics committee recognized by the Israeli authorities for animal experimentation. Stationary organotypic DRG cultures were prepared from rat fetuses (gestational day 15, Lewis inbred, Harlan, Israel). Immediately after dissection, the isolated ganglia were cut with a McIlwain tissue chopper into small slices (of $400 \mu \mathrm{m}$ thickness) which were then seeded in 12 wellculture plates (either directly on the plastic or on glass cover slips) containing $0.75 \mathrm{~mL}$ of NVR-Gel (one explant each well). Cultures received free or conjugated neurotrophic factors (10 ng/mL final concentration, each factor separately) once in the gel at seeding and subsequently with the nutrient medium at each consecutive feeding. Monitoring of the DRG growth pattern (neuronal sprouting, establishment of cultures, and myelin formation) was done by daily phase contrast microscopic observations from 24 hours after setting the cultures onward.

Experiments aimed at examining the effect of neurotrophic factors on early neuronal sprouting; cultures were performed with 12 repetitions ( 12 wells) for each neurotrophic factor. During the first week in culture, ganglia which exhibited nerve outgrowth were counted.

Experiments aimed at examining the effect of neurotrophic factors on early onset and progressionof myelin were performed in triplicate for each neurotrophic factor. Cultures with myelinated fibers, which in phase contrast microscopyappear confined by two dark lines along both sides of the fiber (due to the optical property of the myelin sheaths lipids), were recorded and photographed. The myelinated cultures were fixed for histological and immunocytochemical staining as well as for Transmission Electron Microscopy (TEM).

2.7. Immunofluorescence of Myelinated DRG Cultures. After removal of the culture medium, the DRG cultures were washed with phosphate buffered salt solution (PBS) and fixed in $4 \%$ paraformaldehyde for $15 \mathrm{~min}$ and then washed again with PBS. The fixed cells were permeabilized with $0.1 \%$ of Triton X-100 in PBS and then immunoblocked (to avoid nonspecific staining) with a $1 \%$ bovine serum albumin (BSA) in PBS for $1 \mathrm{~h}$ at room temperature. The specimens were then double incubated with mouse antimyelin basic protein antibodies (MBP, Covance, Nr. SMI 94 R, 1: 250) to visualize the myelin and rabbit antineurofilament antibodies (NF, Novus Biologicals, $1: 500$ ) to visualize the neurite outgrowth. The primary antibodies were diluted in $0.1 \%$ BSA and $0.05 \%$ Tween20 in PBS (diluents buffer) and incubated with the specimens overnight at $4^{\circ} \mathrm{C}$. After rinsing with $0.05 \%$ Tween-20 in PBS (wash buffer), the DRG specimens were incubated for $1 \mathrm{~h}$ at room temperature with the appropriate secondary antibodies: Alexa-Fluor-488-conjugated donkey anti-mouse IgG or Alexa-Fluor-594-conjugated donkey anti-rabbit IgG (Jackson ImmunoResearch, USA, 1: 800 in a diluent buffer). Finally, the samples were rinsed again with wash buffer and mounted with mounting medium (Immco Diagnostics, USA). Myelinated cultures were alternatively stained with Luxol Fast Blue. All of the images were observed with an Olympus IX70 microscope.

2.8. Transmission Electron Microscopy (TEM) Analysis. Organotypic DRG cultures, detached from the glass or the plastic substrates, were fixed in $2.5 \%$ glutaraldehyde, washed in Sorensen phosphate buffer $0.1 \mathrm{M}$ ( $\mathrm{pH} 7.4)$ with $1.5 \%$ sucrose, and then fixed in $2 \%$ osmium tetroxide for $2 \mathrm{~h}$. After dehydration in ethanol, samples were cleared in propylene oxide and embedded in Glauerts' embedding mixture of resins consisting of equal parts of Araldite $\mathrm{M}$ and Araldite Harter, HY 964 (Merck, Darmstadt, Germany), containing $0.5 \%$ of the plasticizer dibutyl phthalate and 1-2\% of the accelerator 964, DY 064 (Merck, Darmstadt, Germany). Transverse cross sections of $70 \mathrm{~nm}$ were obtained using an Ultracut UCT ultramicrotome (Leica, Wetzlar, Germany). Sections were then stained with uranyl acetate and lead citrate and examined by a JEM-1010 transmission electron microscope (JEOL, Tokyo, Japan) equipped with a Mega-View-III digital camera and a Soft-Imaging-System (SIS, Münster, Germany) for the computerized acquisition of the images.

2.9. Statistical Analysis. Statistical analysis was performed by Student's $t$-test. The results are expressed as mean \pm standard deviation (SD). $P<0.05$ was accepted as indicating statistical significance. 


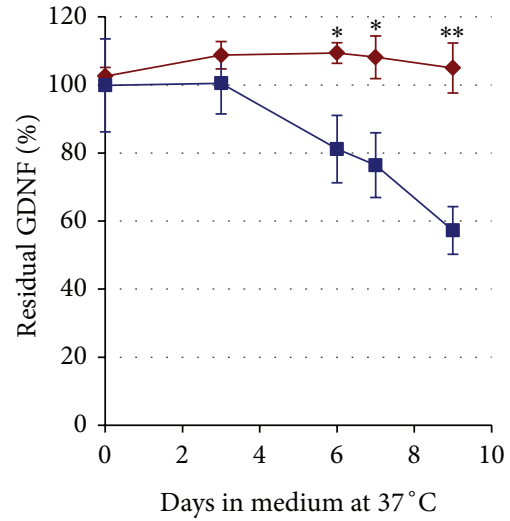

(a1)

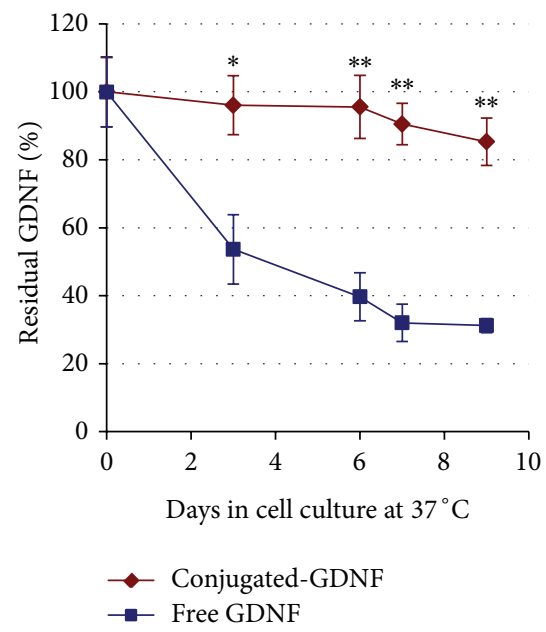

(a2)

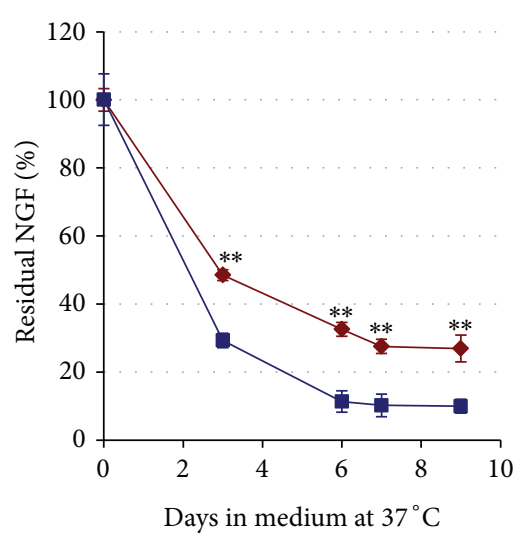

(b1)

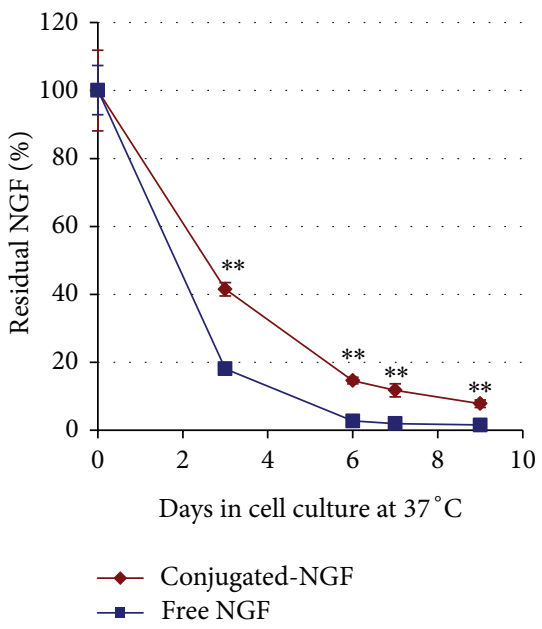

(b2)

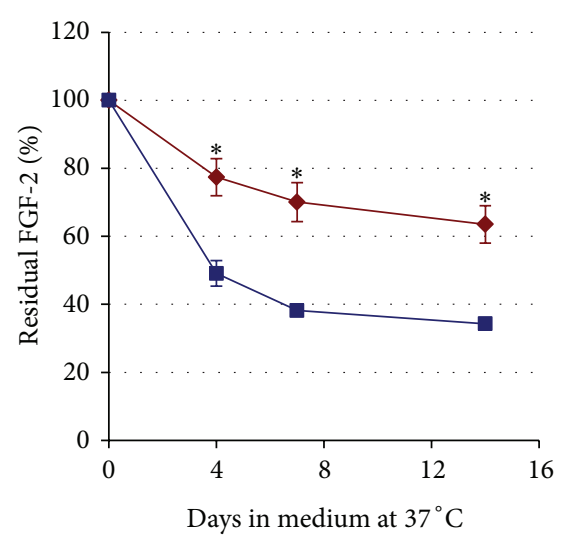

(c1)

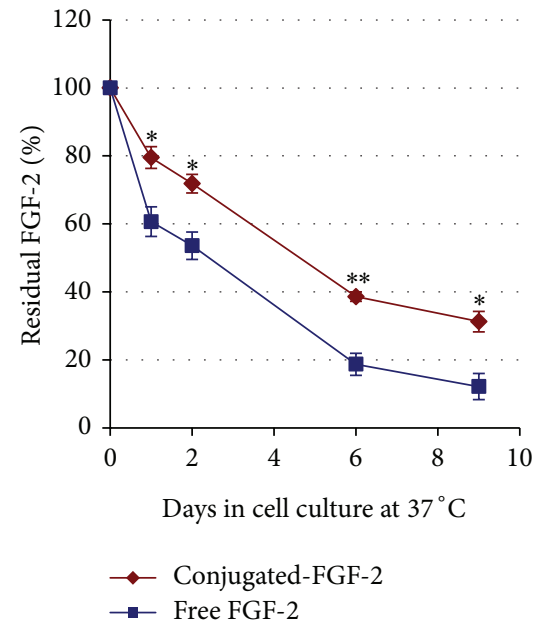

(c2)

FiguRE 1: Stability of free versus conjugated neurotrophic factors at $37^{\circ} \mathrm{C}$ in the absence ((a1), (b1), and (c1)) and in the presence ((a2), (b2), and (c2)) of cells. In the upper row free or conjugated neurotrophic factors (GDNF, $\beta$ NGF, and FGF-2) were added to culture medium, each type separately $\left(10 \mathrm{ng} / \mathrm{mL}\right.$, final concentration), and placed at $37^{\circ} \mathrm{C}$ (in the absence of cells). Aliquots were collected after different time points, and the concentration of the residual factor in the samples was measured using an appropriate ELISA kit. In the lower row, the same concentrations of free or conjugated neurotrophic factors were added once to dissociate dorsal root ganglia (DRG) cell cultures at the beginning of the experiment. The culture medium was not changed during the experiment and aliquots from it were collected at different days after cultivation. The concentration of the residual factors in the aliquots was measured as described above. The data are presented as mean \pm SD in triplicate $(* P<0.01, * * P<0.001)$.

\section{Results}

3.1. Long-Term Stability of Free versus Conjugated Neurotrophic Factors. The stability of the free and conjugated neurotrophic factors (GDNF, $\beta$ NGF, and FGF-2) against various enzymes and inhibitors, existing in serum or secreted from cells, was examined in tissue cultures and in medium alone at $37^{\circ} \mathrm{C}$.

Results shown in Figure 1 demonstrate that the concentration of the neurotrophic factor decreased gradually over time; however, the conjugated neurotrophic factors were significantly more stable than the free factors in both medium alone $((\mathrm{a} 1)-(\mathrm{c} 1))$ and tissue cultures $((\mathrm{a} 2)-(\mathrm{c} 2))$. Among the conjugated factors, the most stable was the conjugated-GDNF ((a1) \& (a2)), followed by the conjugated-FGF-2 with a moderate stability ((c1) \& (c2)), and the least stable was the conjugated- $\beta$ NGF ((b1) \& (b2)). During the 9 days of the experiment, the concentration of the conjugated-GDNF remained almost stable at around $100 \%$, while the concentration of free GDNF was gradually decreased to $57.3 \pm 7.0 \%$ in medium and $31.2 \pm 2.1 \%$ in culture. Regarding the stability of FGF-2, after about a week, the residual concentrations of the conjugated-FGF-2 in medium and in tissue culture were reduced to $70.1 \pm 5.7 \%$ and $38.6 \pm 1.4 \%$, respectively. On the other hand, the residual concentrations of the free FGF-2 were significantly lower than that of the conjugated factor: $38.2 \pm 1.6 \%$ and $18.7 \pm 3.2 \%$, in medium and in the tissue culture, respectively. As to the $\beta \mathrm{NGF}$, after 6 days, the residual concentrations of the conjugated- $\beta$ NGF were reduced to $32.6 \pm 2.0 \%$ in medium and $14.6 \pm 1.0 \%$ in culture. 


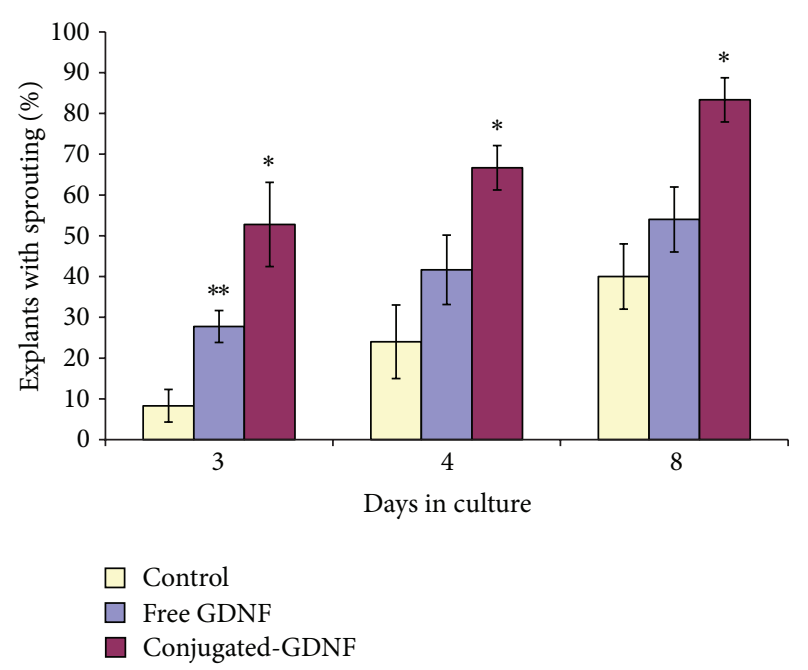

FIGURE 2: Enhancement of early nerve fibre outgrowth by conjugated-GDNF. The graph shows the percentage of explants (out of 12) which exhibited nerve fiber sprouting in the absence and the presence of free versus conjugated-GDNF as a function of time (days) in culture. The data are presented as mean \pm SD from 3 repeated experiments. $*$ Significant difference $(P<0.05)$ compared to the corresponding free group. $* *$ Significant difference $(P<0.05)$ compared to the corresponding control group.

However, the free $\beta$ NGF was decreased more significantly to $14.6 \pm 1.0 \%$ in medium and $2.8 \pm 1.5 \%$ in tissue culture.

3.2. Effects of Free versus Conjugated Neurotrophic Factors on DRG Organotypic Cultures. The overall effect of the exposure of DRG explants to each of the three conjugated neurotrophic factors is represented by the early phenomenon of sprouting compared to cultures that were exposed to free factors and control. During the first days of cultivation, the number of DRG explants that exhibited an early sprouting, following the exposure to conjugatedGDNF, was increased significantly by $30 \%$ compared to free GDNF and by $40 \%$ compared to controls (without neurotrophic factors) (Figure 2). The most intensive sprouting was observed in cultures which were exposed to $\beta$ NGF and GDNF (Figure 3). During the establishment of the cultures (after the first week of cultivation), the regenerated nerve fibers became longer, thicker, and ramified, until the formation of neuronal networks, mainly in cultures exposed to conjugated-GDNF and $\beta \mathrm{NGF}$ (Figure 4). However, only conjugated-GDNF accelerated significantly the onset of myelin.

The effect of free versus conjugated neurotrophic factors on the onset of myelin in organotypic DRG cultures is shown in Figure 5. The results demonstrate that the onset of myelin (the appearance of initial segments of myelinated axons) was observed first in cultures exposed to conjugated-GDNF. In these cultures, onset of myelin was already observed after $14 \pm 2.2$ days of cultivation. Furthermore, during subsequent days, most of the fibers in cultures incubated with conjugatedGDNF became heavily myelinated (Figure 6). In contrast, the onset of myelin in cultures exposed to free GDNF was observed significantly later, that is, after $20.8 \pm 4.3$ days of cultivation ( $P=0.0006$, compared to conjugatedGDNF). The onset of myelin in cultures exposed to free and conjugated- $\beta$ NGF was observed only after more than 3 weeks of cultivation (on days $27.3 \pm 2.9$ and $23.8 \pm$ 4.0 , resp.). Although in these cultures the onset of myelin was advanced $(P<0.05)$ in comparison to control cultures (without neurotrophic factors), yet it was significantly decelerated $(P<0.001)$ compared to cultures exposed to conjugated-GDNF. The onset of myelin in cultures exposed to free and conjugated-FGF-2 was observed around the fourth week in culture, on days $29.3 \pm 3.2$ and $27.3 \pm$ 6.0 , respectively, which demonstrates a nonsignificant $(P>$ 0.05 ) acceleration compared to controls, in which the phenomenon occurred $34.0 \pm 4.2$ days after cultivation. Results similar to those obtained from control cultures were also obtained in cultures which were exposed to naked iron oxide nanoparticles.

3.3. TEM Analysis. TEM analysis was made on early myelinated DRG organotypic cultures (14 days after seeding) performed in NVR-Gel enriched with conjugated-GDNF. TEM observations (Figure 7) confirmed the light microscopic observations regarding the existence of myelin around the nerve fibers. However, TEM analysis allowed further observation of the very small single iron oxide nanoparticles and to determine their localization. In Figure 7, a transverse section ((a) and (b)) and a longitudinal section ((c) and (d)) of a nerve fiber with its myelin sheaths are shown. The ultrastructural analysis allowed us to observe single/distinct iron oxide nanoparticles (of average size of around 10-15 nm) localized between the ensheathing myelin lamellae (black arrows) and within the axon (white arrows). These TEM observations clearly demonstrate that the GDNF-conjugated iron oxide nanoparticles were internalized by neurons and by Schwann cells.

\section{Discussion}

The present study describes a novel strategy for DRG cultivation in NVR-Gel containing neurotrophic factors ( $\beta \mathrm{NGF}$, GDNF, and FGF-2) covalently conjugated to iron oxide nanoparticles. The neurotrophic factors were stabilized by the covalent conjugation to the iron oxide nanoparticles. This was demonstrated by comparative stability studies (Figure 1) in which the conjugated neurotrophic factors were significantly more stable than the free factors in cell cultures and medium at $37^{\circ} \mathrm{C}$. The increased stability of the conjugated neurotrophic factors is probably due to the fact that the coupling of proteins to nanoparticles protects them from proteolytic enzymes and inhibitors existing in the serum or secreted by the cells and thus prolongs their half-life time and enhances their activity in vitro and in vivo, as described previously [21, 22, 33, 35-39].

The biological activity of the neurotrophic factors was examined in cultures prepared in NVR-Gel. The results showed that the gel adhered easily the DRG slices to the glass or plastic substrates and subsequently supported cell 


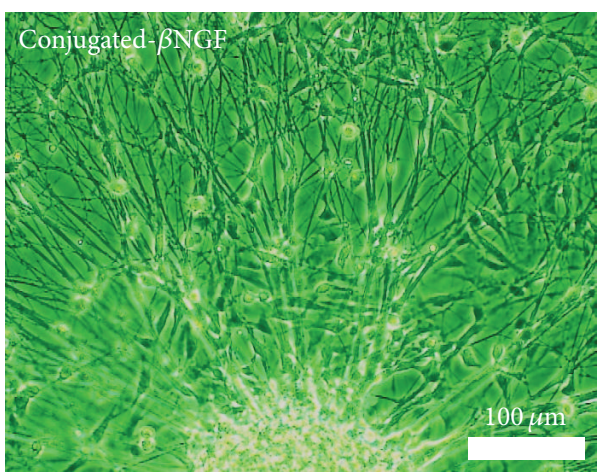

(a)

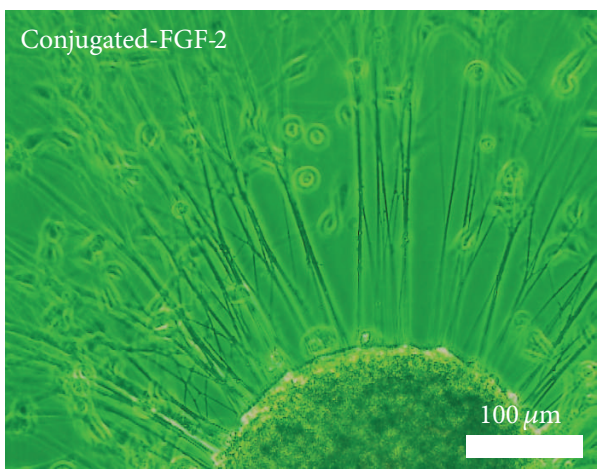

(c)

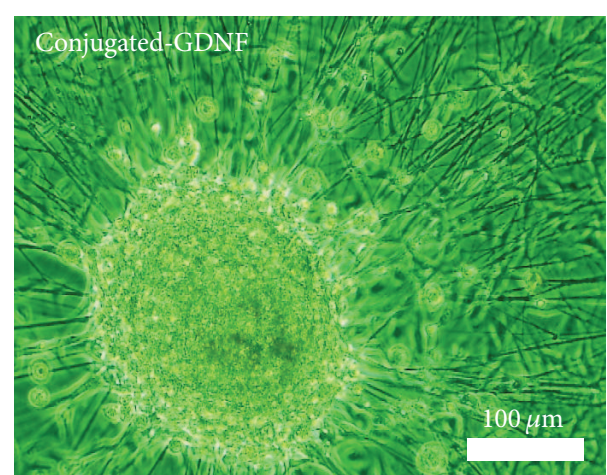

(b)

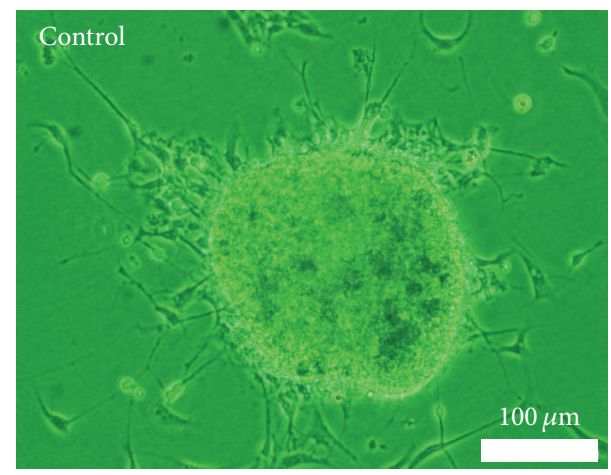

(d)

FIGURE 3: Early sprouting of thin and long nerve fibers from DRG explants $24 \mathrm{~h}$ after cultivation in NVR-Gel enriched with growth factors conjugated to iron oxide nanoparticles as follows: (a) Conjugated- $\beta$ NGF, (b) Conjugated-GDNF, (c) Conjugated-FGF-2, (d) Control (without growth factors) (phase-contrast microscopy).

maturation and nerve fiber outgrowth in a three-dimensional pattern. Similar properties of the gel have been demonstrated previously, both in neuronal cell cultures [21, 40] and in in vivo models of peripheral nerve injuries and spinal cord injuries [41, 42].

Comparative studies on the biological effect of free versus conjugated- $\beta$ NGF, -GDNF, and -FGF- 2 on DRG cultures revealed that during the first days of cultivation the conjugated neurotrophic factors had more beneficial effects than the corresponding free neurotrophic factors on the enhancement of early nerve fiber regeneration (Figure 2). In the long-term cultures, the conjugated-GDNF had the most significant efficacy (Figure 5), which was expressed in acceleration of the myelin onset and subsequently the progression of myelination. This is probably due to covalent binding of the GDNF to the iron oxide nanoparticles, which increases the conjugated neurotrophic factor stability and prolongs its biological activity. As far as we know, there are no other publications who report that GDNF conjugated to nanoparticles, of any kind, enhances onset and progression of myelin. Free GDNF had a significantly reduced effect in comparison to the conjugated-GDNF.

The difference in the effect of conjugated-GDNF and conjugated- $\beta$ NGF on the acceleration of myelin formation in DRG cultures is intriguing since both conjugated factors had a similar beneficial effect on early neurite outgrowth and formation of axonal networks. Since myelin of the peripheral nervous system is known to be formed only around axons that have a diameter of $2 \mu \mathrm{m}$ and more, we expected that myelin will equivalently form around the thick axons in cultures incubated with either of neurotrophic factors. However, the conjugated-GDNF accelerated the early onset and progression of myelin significantly over the conjugated- $\beta$ NGF $(P<0.0001)$ (Figure 5). This strongly indicates that conjugated-GDNF, but not the $\beta$ NGF, has an additional beneficial effect on Schwann cells, which results in the enhancement of the peripheral myelin formation.

Indeed, the TEM images (Figure 7) clearly demonstrate that a large number of iron oxide nanoparticles conjugated to GDNF were internalized by neurons as well as by Schwann cells. These results strongly support the hypothesis that GDNF activates both on DRG neurons and Schwann cells. This hypothesis is strengthened by Zhang et al. [11] who reported that free GDNF, beside its effect on enhancement of axonal regeneration in vivo and in vitro, significantly increases the number of myelin sheaths produced by Schwann cells. Similarly, Höke et al. [10] found that administration of free GDNF to adult rats alters axon-Schwann cell units and promotes myelination of unmyelinated nerve fibers. 


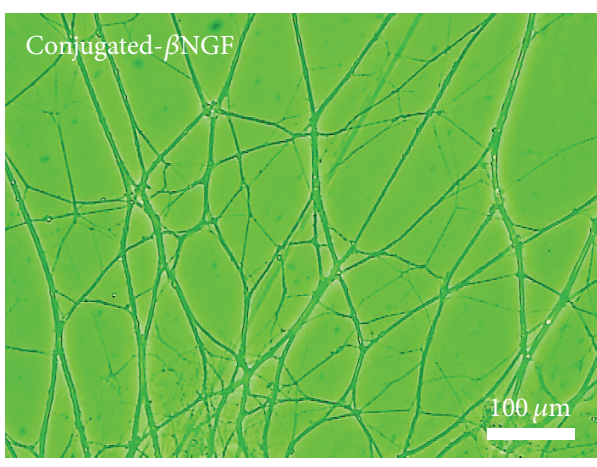

(a)

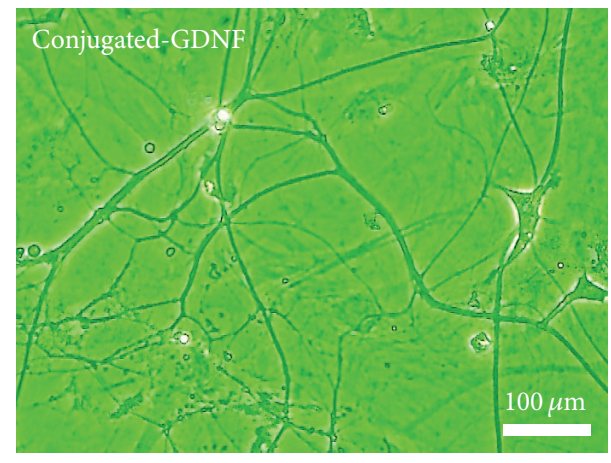

(b)

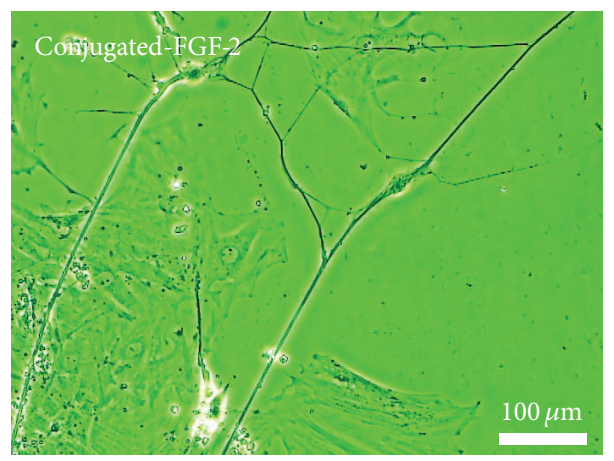

(c)

FIGURE 4: Establishment of cultures: neuronal networks composed of long, thick, and ramified neurites which are originating from DRG explants cultures, 10 days after set-up in NVR-Gel enriched with neurotrophic factors conjugated to iron oxide nanoparticles as follows: (a) Conjugated- $\beta$ NGF, (b) Conjugated-GDNF, (c) Conjugated-FGF-2 (phase-contrast microscopy).

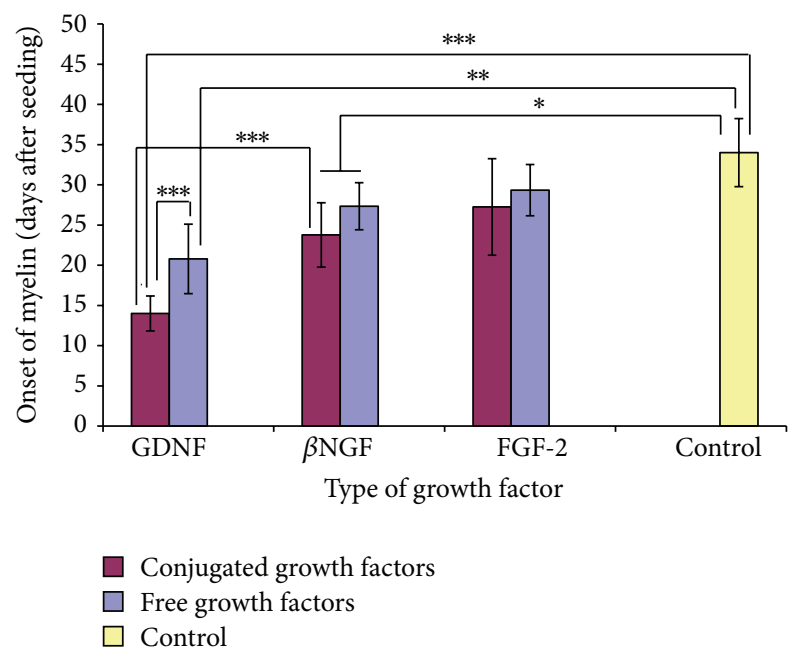

FIGURE 5: Effect of neurotrophic factors conjugated to iron oxide nanoparticles versus free factors on the onset of myelin in DRG organotypic cultures. The graph shows time elapsed (days) until the appearance of the first segments of myelinated axons. Control cultures have not been exposed to neurotrophic factors. The data represented shows mean \pm SD from 15 repeated experiments. In each experiment cultures were performed in triplicate for each type of neurotrophic factor $(* P<0.05, * * P<0.01, * * * P<0.001)$.

\section{Conclusion}

These studies demonstrate that the covalent conjugation of neurotrophic factors to iron oxide nanoparticles increases their stability, preserves their activity, and even improves it. Comparative biological studies performed on DRG cultures in NVR-Gel revealed that the gel enriched with conjugated$\beta$ NGF, -GDNF, and -FGF-2 induced early nerve fiber regeneration significantly before the corresponding free neurotrophic factors. Among the three conjugated neurotrophic factors, the conjugated-GDNF had the most meaningful effect on the early onset and the progression of myelin in DRG cultures. The onset of myelin in cultures enriched with conjugated-GDNF was detected 7 days earlier than in cultures incubated with free GDNF and 21 days earlier than in control cultures.

Our culture strategy, which combines the employment of NVR-Gel and stabilized conjugated-GDNF, reveals that the biomaterials evaluated could become a scaffold material for a bioartificial nerve graft for long gap peripheral nerve reconstruction. Such an implant will most likely enhance regeneration and myelin formation of the damaged axons and thus has potential to shorten the recovery period. The organotypic DRG cultures in NVR-Gel enriched with conjugated factors can also serve as an in vitro pharmacological model for the research of demyelination and 


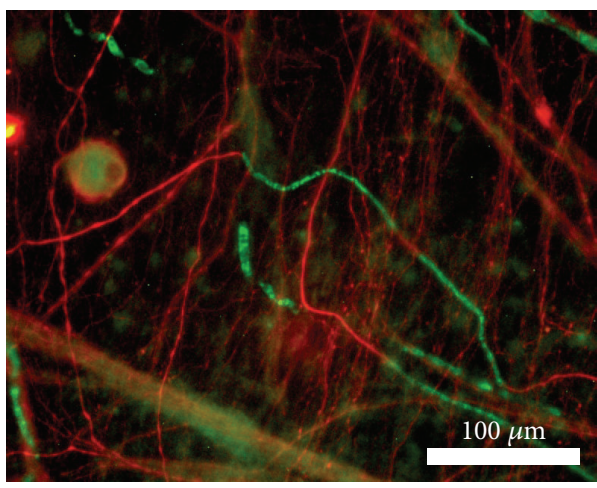

(a)

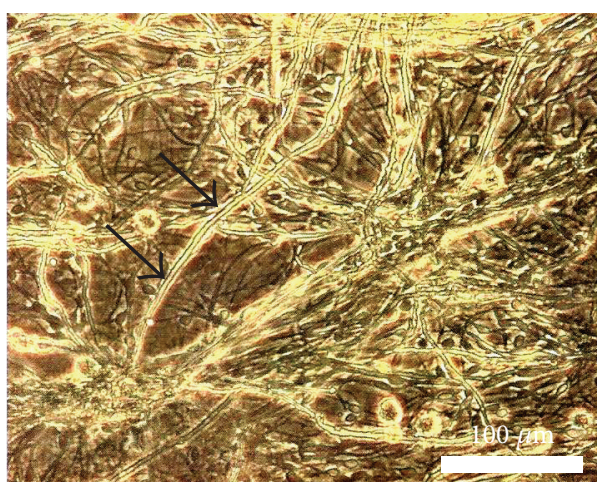

(c)

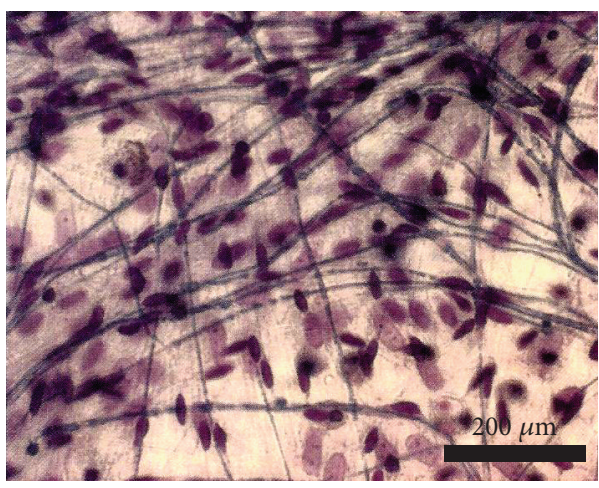

(e)

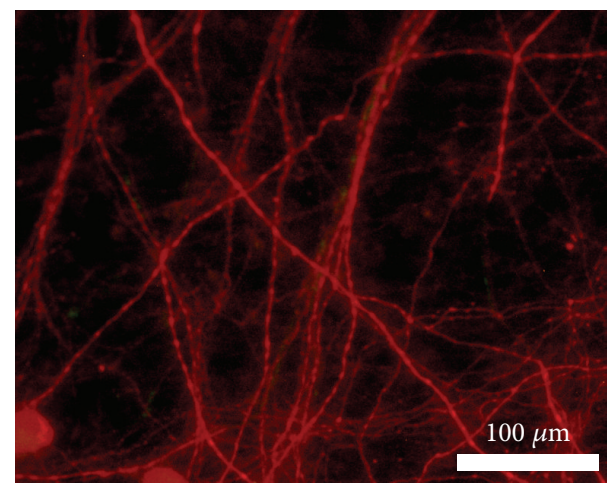

(b)

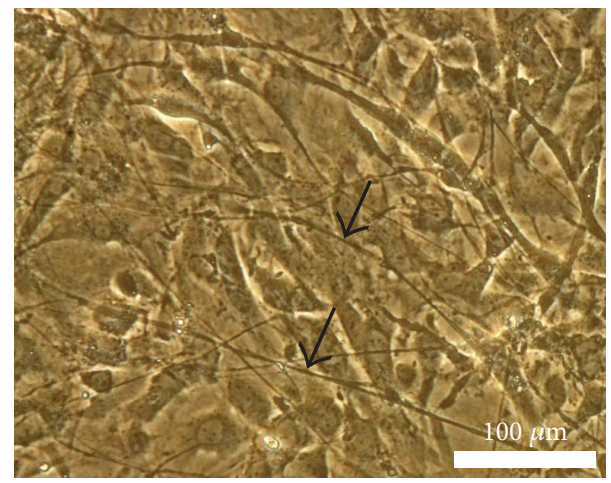

(d)

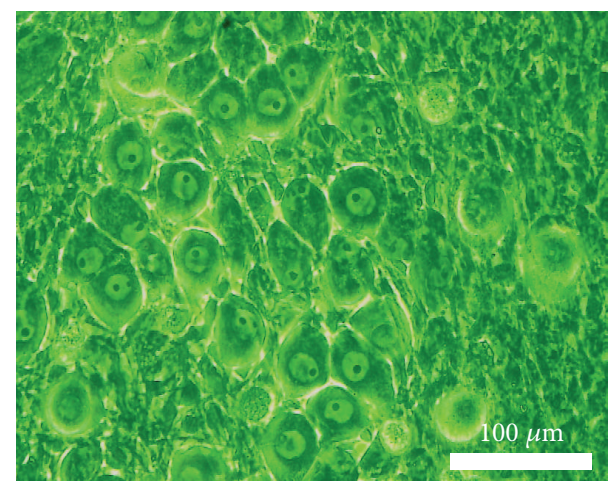

(f)

FIGURE 6: Myelinated organotypic DRG cultures exposed to conjugated-GDNF (left column) versus nonmyelinated cultures (right column). (a) and (b): Representative immunofluorescence images of a myelinated (day 12) versus nonmyelinated cultures, respectively. Neurites appear in red (antineurofilament staining) and myelin sheaths appear in green (antimyelin basic protein staining). (c) and (d): Phase-contrast microscope images of a heavily myelinated (day 16) versus nonmyelinated culture, respectively. Note the arrows in (c) which point to a single birefringent myelinated nerve fibre (appears as a bright fibre confined by two dark lines along both its sides due to the optical property of the myelin sheaths lipids). The arrows in (d) point to nonmyelinated fibres. (e): Staining of myelinated nerve fibres with Luxol Fast Blue. (f): Phase-contrast microscope image (through a green filter) of individual DRG cells in a nonmyelinated culture.

remyelination in autoimmune diseases and in bacterial and viral intoxications.

\section{Conflict of Interests}

The authors declare that there is no conflict of interests regarding the publication of this paper.

\section{Acknowledgments}

Part of this work has received funding from the European Community's Seventh Framework Program (FP7-HEALTH2011) under Grant Agreement no. 278612 (BIOHYBRID). The authors gratefully acknowledge Stefania Raimondo and Giulia Ronchi members of Università di Torino for their help with the TEM analysis. 


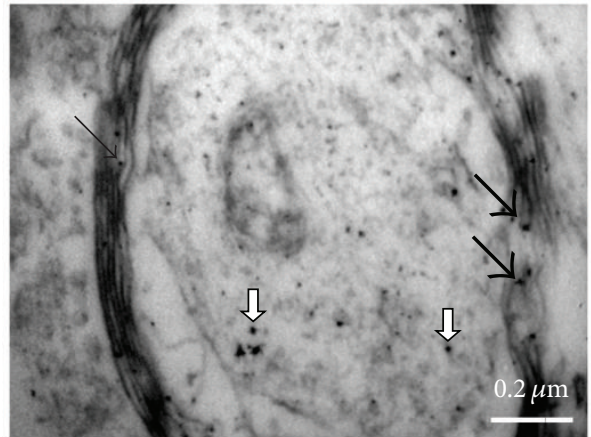

(a)

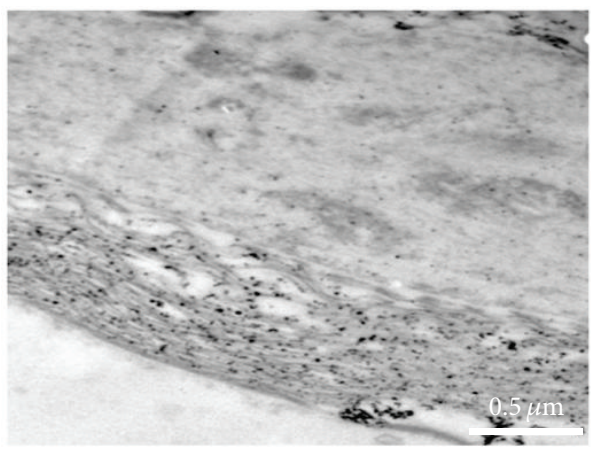

(c)

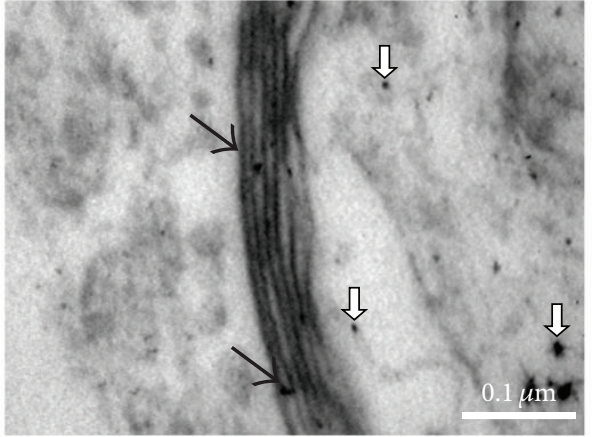

(b)

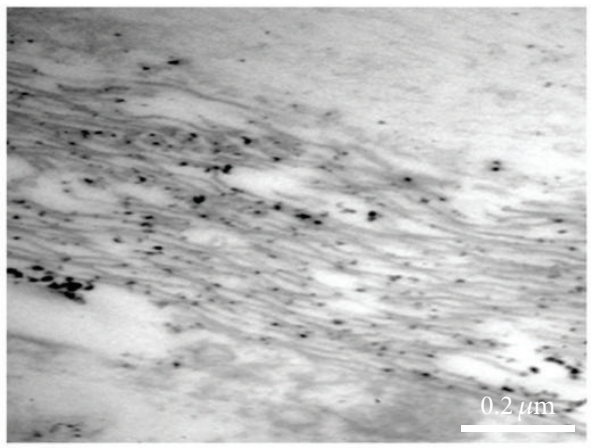

(d)

FIGURE 7: TEM analysis of the localisation of GDNF-conjugated iron oxide nanoparticles: Myelinated organotypic DRG cultures 14 days after seeding in NVR-Gel containing GDNF-conjugated nanoparticles were analysed. (a) and (b): Axon in transverse section. (c) and (d): Axon in longitudinal section. The black arrows point to iron oxide nanoparticles between myelin lamellae and the white arrows point to iron oxide nanoparticles in the axon.

\section{References}

[1] G. Lundborg, "Alternatives to autologous nerve grafts," Handchirurgie, Mikrochirurgie, Plastische Chirurgie, vol. 36, no. 1, pp. $1-7,2004$.

[2] C. Grothe, K. Haastert-Talini, T. Freier et al., "BIOHYBRIDBiohybrid templates for peripheral nerve regeneration," Journal of the Peripheral Nervous System, vol. 17, no. 2, pp. 220-222, 2012.

[3] Y.-C. Huang and Y.-Y. Huang, "Tissue engineering for nerve repair," Biomedical Engineering: Applications, Basis and Communications, vol. 18, pp. 100-110, 2006.

[4] S. M. Willerth and S. E. Sakiyama-Elbert, "Approaches to neural tissue engineering using scaffolds for drug delivery," Advanced Drug Delivery Reviews, vol. 59, no. 4-5, pp. 325-338, 2007.

[5] K. S. Straley, C. W. P. Foo, and S. C. Heilshorn, "Biomaterial design strategies for the treatment of spinal cord injuries," Journal of Neurotrauma, vol. 27, no. 1, pp. 1-19, 2010.

[6] P. Lu and M. H. Tuszynski, "Growth factors and combinatorial therapies for CNS regeneration," Experimental Neurology, vol. 209, no. 2, pp. 313-320, 2008.

[7] L. Aloe, "Rita Levi-Montalcini: the discovery of nerve growth factor and modern neurobiology," Trends in Cell Biology, vol. 14, no. 7, pp. 395-399, 2004.

[8] S. Varon, J. Nomura, and E. M. Shooter, "Reversible dissociation of the mouse nerve growth factor protein into different subunits," Biochemistry, vol. 7, no. 4, pp. 1296-1303, 1968.

[9] W. Sun, C. Sun, H. Lin et al., "The effect of collagen-binding NGF- $\beta$ on the promotion of sciatic nerve regeneration in a rat sciatic nerve crush injury model," Biomaterials, vol. 30, no. 27, pp. 4649-4656, 2009.

[10] A. Höke, T. Ho, T. O. Crawford, C. LeBel, D. Hilt, and J. W. Griffin, "Glial cell line-derived neurotrophic factor alters axon Schwann cell units and promotes myelination in unmyelinated nerve fibers," The Journal of Neuroscience, vol. 23, no. 2, pp. 561567, 2003.

[11] L. Zhang, Z. Ma, G. M. Smith et al., "GDNF-enhanced axonal regeneration and myelination following spinal cord injury is mediated by primary effects on neurons," GLIA, vol. 57 , no. 11, pp. 1178-1191, 2009.

[12] K. D. Beck, J. Valverde, T. Alexi et al., "Mesencephalic dopaminergic neurons protected by GDNF from axotomy-induced degeneration in the adult brain," Nature, vol. 373, no. 6512, pp. 339-341, 1995.

[13] S. B. Rangasamy, K. Soderstrom, R. A. E. Bakay, and J. H. Kordower, "Neurotrophic factor therapy for Parkinson's disease," Progress in Brain Research, vol. 184, pp. 237-264, 2010.

[14] R. S. Morrison, A. Sharma, J. de Vellis, and R. A. Bradshaw, "Basic fibroblast growth factor supports the survival of cerebral cortical neurons in primary culture," Proceedings of the National Academy of Sciences of the United States of America, vol. 83, no. 19, pp. 7537-7541, 1986.

[15] A. Bikfalvi, S. Klein, G. Pintucci, and D. B. Rifkin, "Biological roles of fibroblast growth factor-2," Endocrine Reviews, vol. 18, no. 1, pp. 26-45, 1997. 
[16] C. Grothe and G. Nikkhah, "The role of basic fibroblast growth factor in peripheral nerve regeneration," Anatomy and Embryology, vol. 204, no. 3, pp. 171-177, 2001.

[17] I. Allodi, L. Casals-Díaz, E. Santos-Nogueira, F. GonzalezPerez, X. Navarro, and E. Udina, "FGF-2 low molecular weight selectively promotes neuritogenesis of motor neurons in vitro," Molecular Neurobiology, vol. 47, no. 2, pp. 770-781, 2013.

[18] K. Haastert, E. Lipokatić, M. Fischer, M. Timmer, and C. Grothe, "Differentially promoted peripheral nerve regeneration by grafted Schwann cells over-expressing different FGF-2 isoforms," Neurobiology of Disease, vol. 21, no. 1, pp. 138-153, 2006.

[19] C. Grothe, K. Haastert, and J. Jungnickel, "Physiological function and putative therapeutic impact of the FGF-2 system in peripheral nerve regeneration-lessons from in vivo studies in mice and rats," Brain Research Reviews, vol. 51, no. 2, pp. 293299, 2006.

[20] B. Knusel, P. P. Michel, J. S. Schwaber, and F. Hefti, "Selective and nonselective stimulation of central cholinergic and dopaminergic development in vitro by nerve growth factor, basic fibroblast growth factor, epidermal growth factor, insulin and the insulinlike growth factors I and II," Journal of Neuroscience, vol. 10, no. 2, pp. 558-570, 1990.

[21] H. Skaat, O. Ziv-Polat, A. Shahar, and S. Margel, "Enhancement of the growth and differentiation of nasal olfactory mucosa cells by the conjugation of growth factors to functional nanoparticles," Bioconjugate Chemistry, vol. 22, no. 12, pp. 2600-2610, 2011.

[22] O. Ziv-Polat, H. Skaat, A. Shahar, and S. Margel, "Novel magnetic fibrin hydrogel scaffolds containing thrombin and growth factors conjugated iron oxide nanoparticles for tissue engineering," International Journal of Nanomedicine, vol. 7, no. 1, pp. 1259-1274, 2012.

[23] D. Schubert, "Synergistic interactions between transforming growth factor beta and fibroblast growth factor regulate Schwann cell mitosis," Journal of Neurobiology, vol. 23, no. 2, pp. 143-148, 1992.

[24] S. Zhang and H. Uludağ, "Nanoparticulate systems for growth factor delivery," Pharmaceutical Research, vol. 26, no. 7, pp. 15611580, 2009.

[25] E. R. Edelman, M. A. Nugent, and M. J. Karnovsky, "Perivascular and intravenous administration of basic fibroblast growth factor: Vascular and solid organ deposition," Proceedings of the National Academy of Sciences of the United States of America, vol. 90, no. 4, pp. 1513-1517, 1993.

[26] G. F. Whalen, Y. Shing, and J. Folkman, "The fate of intravenously administered bFGF and the effect of heparin," Growth Factors, vol. 1, no. 2, pp. 157-164, 1989.

[27] T. Sakane and W. M. Pardridge, "Carboxyl-directed pegylation of brain-derived neurotrophic factor markedly reduces systemic clearance with minimal loss of biologic activity," Pharmaceutical Research, vol. 14, no. 8, pp. 1085-1091, 1997.

[28] D. N. Ishii and E. M. Shooter, "Regulation of nerve growth factor synthesis in mouse submaxillary glands by testosterone," Journal of Neurochemistry, vol. 25, no. 6, pp. 843-851, 1975.

[29] P. Pradier, I. Jalenques, M. Dalle, R. Reuling, G. Despres, and R. Romand, "Distribution and metabolism patterns of plasma 7Sand $\beta$-NGF in the adult male rat," Journal of Physiology Paris, vol. 88 , no. 5 , pp. 273-277, 1994.

[30] K. Lee, E. A. Silva, and D. J. Mooney, "Growth factor deliverybased tissue engineering: general approaches and a review of recent developments," Journal of the Royal Society Interface, vol. 8, no. 55, pp. 153-170, 2011.
[31] C. C. Berry and A. S. G. Curtis, "Functionalisation of magnetic nanoparticles for applications in biomedicine," Journal of Physics D Applied Physics, vol. 36, no. 13, pp. R198-R206, 2003.

[32] A. Shahar, Z. Nevo, and S. Rochkind, "Cross-linked hyaluronic acid-laminin gels and use thereof in cell culture and medical implants," US Patent 0024373, 2001.

[33] S. Margel, O. Ziv-Polat, H. Skaat, A. Shahar, and Assignee BarIlan University, "Novel nanocomposite gel and fibrin scaffold for cell implantation and tissue engineering," Provisional US Patent Application no. 61457989 (7/2011), 61678109 (8/2012), 2012.

[34] R. S. Molday and D. Mackenzie, "Immunospecific ferromagnetic iron-dextran reagents for the labeling and magnetic separation of cells," Journal of Immunological Methods, vol. 52, no. 3, pp. 353-367, 1982.

[35] H. Skaat, O. Ziv-Polat, A. Shahar, and S. Margel, "Enhanced cell growth by new magnetic scaffolds containing bioactiveconjugated nanoparticles for tissue engineering," in Applications of Nanomaterials, J. N. Govil, Ed., Studium Press LLC, Houston, Tex, USA, 2012.

[36] T. Green-Sadan, Y. Kuttner, T. Lublin-Tennenbaum et al., "Glial cell line-derived neurotrophic factor-conjugated nanoparticles suppress acquisition of cocaine self-administration in rats," Experimental Neurology, vol. 194, no. 1, pp. 97-105, 2005.

[37] O. Ziv-Polat, M. Topaz, T. Brosh, and S. Margel, "Enhancement of incisional wound healing by thrombin conjugated iron oxide nanoparticles," Biomaterials, vol. 31, no. 4, pp. 741-747, 2010.

[38] G. Shafir, A. Galperin, and S. Margel, "Synthesis and characterization of recombinant factor VIIa-conjugated magnetic iron oxide nanoparticles for hemophilia treatment," Journal of Biomedical Materials Research A, vol. 91, no. 4, pp. 1056-1064, 2009.

[39] E. Corem-Salkmon, Z. Ram, D. Daniels et al., "Convectionenhanced delivery of methotrexate-loaded maghemite nanoparticles," International Journal of Nanomedicine, vol. 6, pp. 1595-1602, 2011.

[40] S. Rochklnd, D. El-Ani, Z. Nevo, and A. Shahar, "Increase of neuronal sprouting and migration using $780 \mathrm{~nm}$ laser phototherapy as procedure for cell therapy," Lasers in Surgery and Medicine, vol. 41, no. 4, pp. 277-281, 2009.

[41] S. Rochkind, A. Shahar, D. Fliss et al., "Development of a tissueengineered composite implant for treating traumatic paraplegia in rats," European Spine Journal, vol. 15, no. 2, pp. 234-245, 2006.

[42] S. Rochkind, L. Astachov, D. El-Ani et al., "Further development of reconstructive and cell tissue-engineering technology for treatment of complete peripheral nerve injury in rats," Neurological Research, vol. 26, no. 2, pp. 161-166, 2004. 

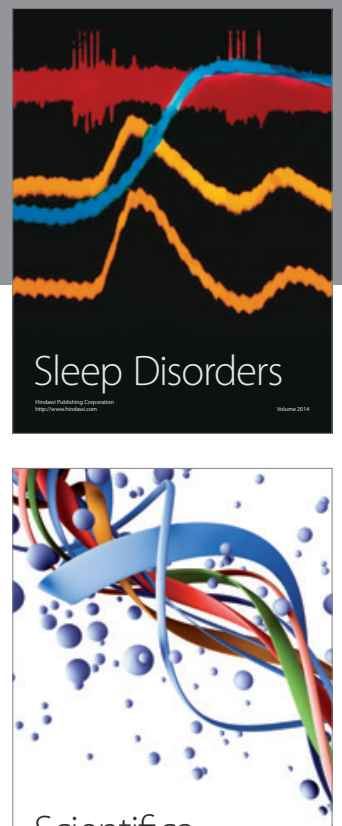

Scientifica
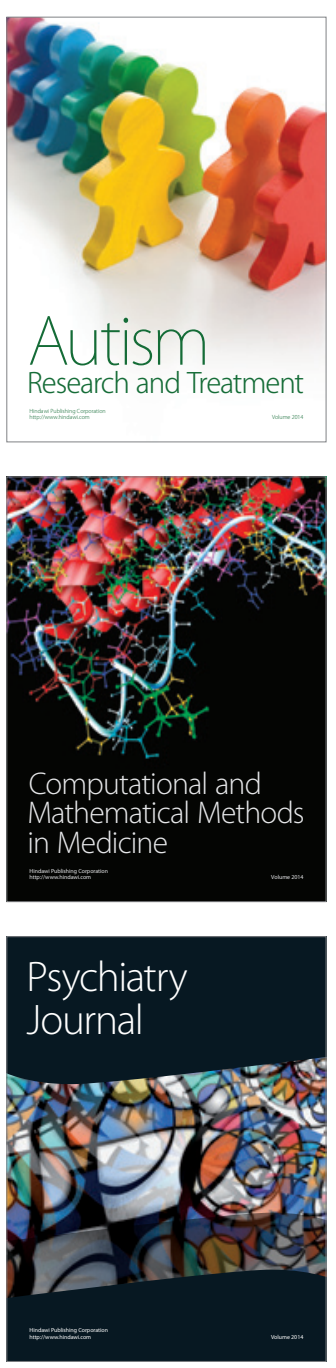
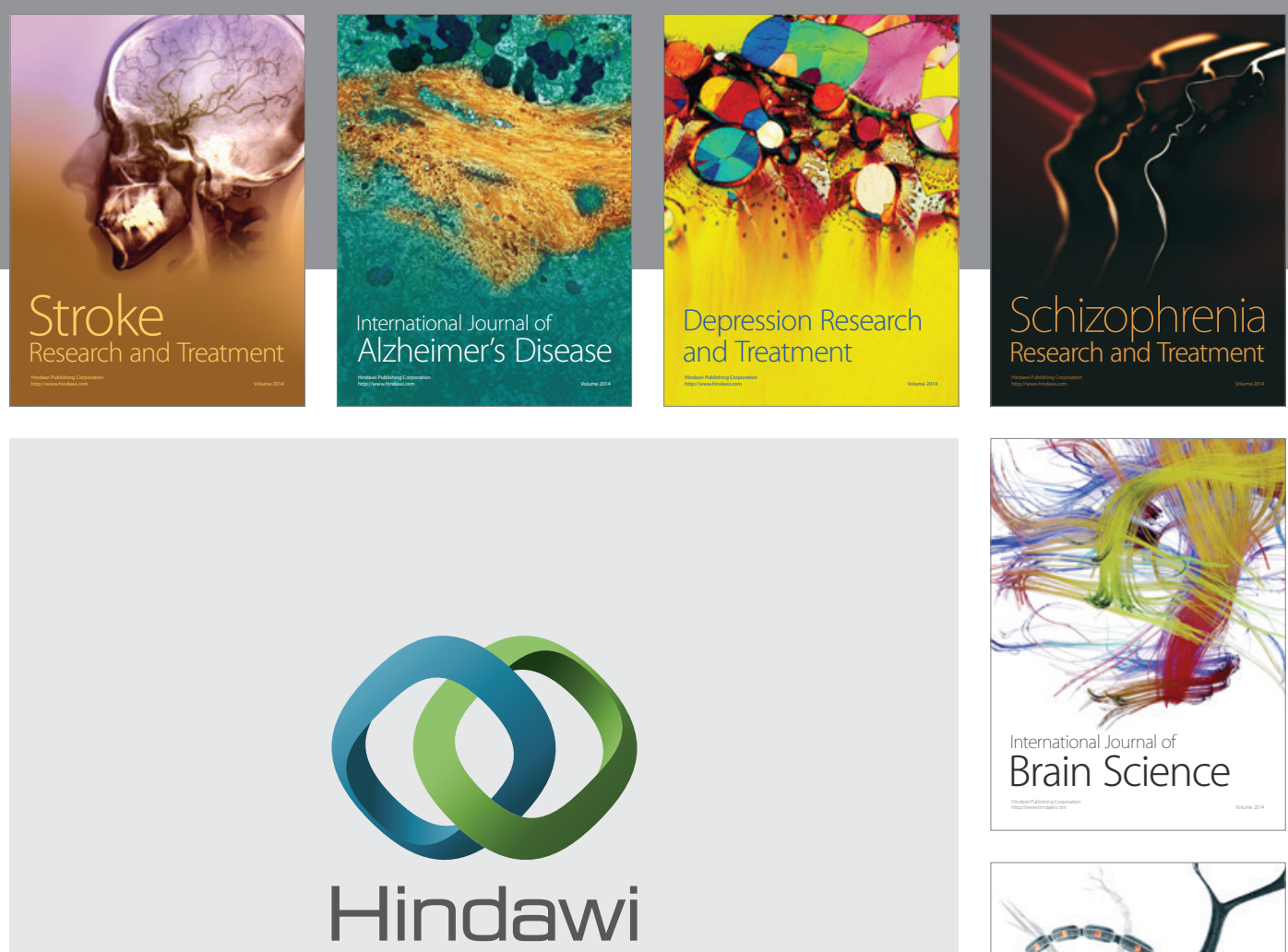

Submit your manuscripts at

http://www.hindawi.com
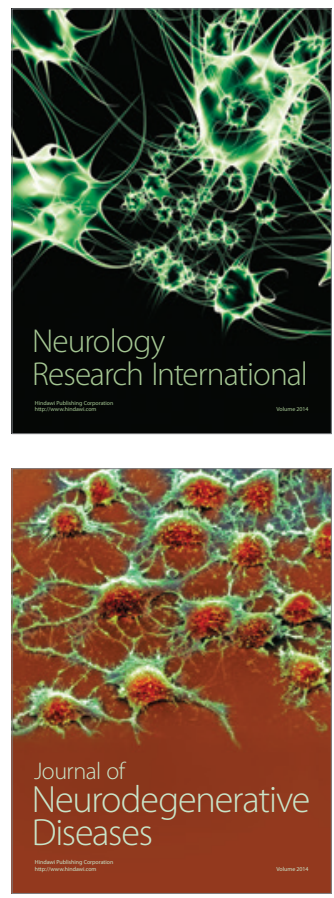

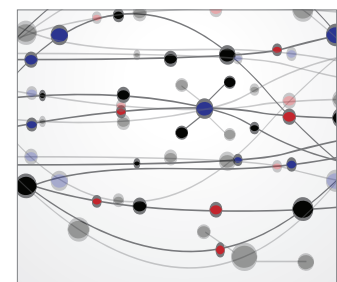

The Scientific World Journal
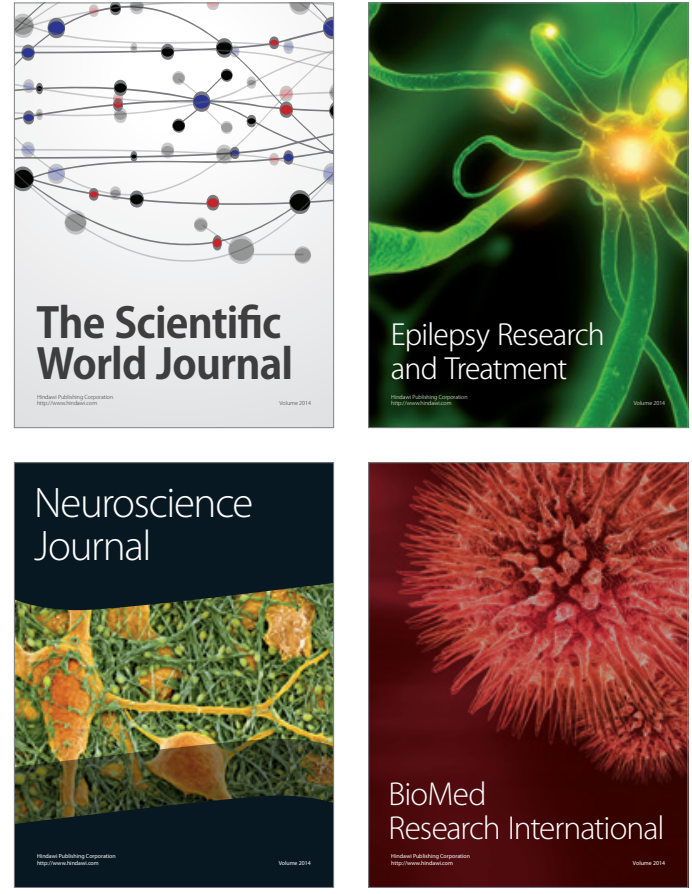

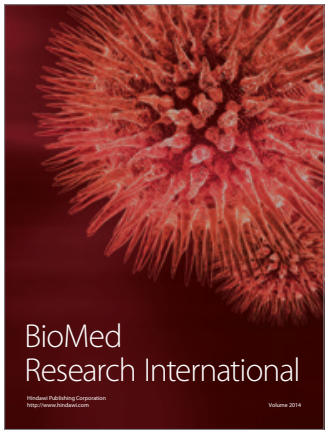

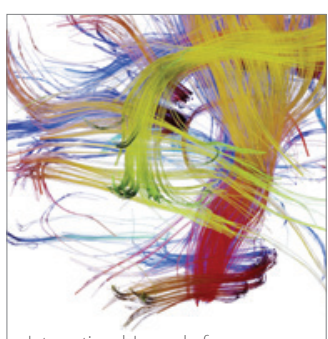

Brain Science

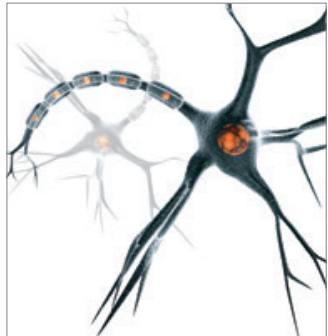

Neural Plasticity
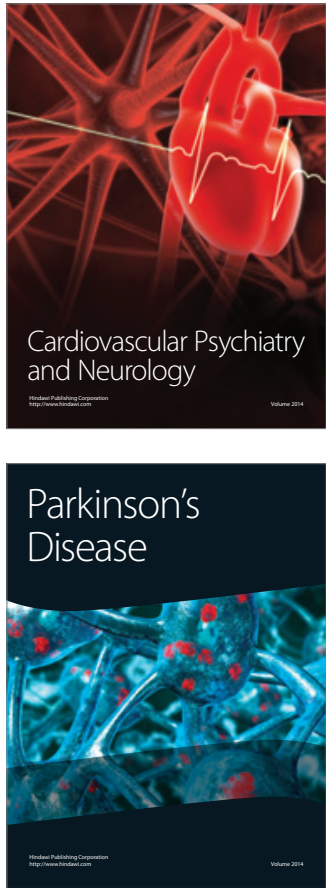